\title{
Grey Matter Volume in the Cerebellum is Related to the Processing of Grammatical Rules in a Second Language: A Structural Voxel-based Morphometry Study
}

\author{
Christos Pliatsikas • Tom Johnstone • Theodoros Marinis \\ Published online: 30 August 2013 \\ (C) The Author(s) 2013. This article is published with open access at Springerlink.com
}

\begin{abstract}
The experience of learning and using a second language (L2) has been shown to affect the grey matter (GM) structure of the brain. Importantly, GM density in several cortical and subcortical areas has been shown to be related to performance in L2 tasks. Here, we show that bilingualism can lead to increased GM volume in the cerebellum, a structure that has been related to the processing of grammatical rules. Additionally, the cerebellar GM volume of highly proficient L2 speakers is correlated to their performance in a task tapping on grammatical processing in an L2, demonstrating the importance of the cerebellum for the establishment and use of grammatical rules in an L2.
\end{abstract}

Keywords Cerebellum · VBM · Grammatical processing · Second language

\footnotetext{
C. Pliatsikas $(\bowtie) \cdot$ T. Johnstone

Centre for Integrative Neuroscience and Neurodynamics, University of Reading, Harry Pitt Building, Whiteknights, RG6 6AL Reading, Berkshire, UK

e-mail: c.pliatsikas@reading.ac.uk

T. Johnstone

e-mail: i.t.johnstone@reading.ac.uk

C. Pliatsikas · T. Marinis

Department of Clinical Language Sciences, School of Psychology and Clinical Language Sciences, University of Reading, RG6 6AL Reading, UK

T. Marinis

e-mail: t.marinis@reading.ac.uk

T. Johnstone

Department of Psychology, School of Psychology and Clinical

Language Sciences, University of Reading, RG6 6AL Reading, UK
}

\section{Introduction}

In an influential paper on the memory circuits that subserve language processing, Ullman [1] developed the declarative/ procedural (DP) model. According to this approach, there is a distinction in the brain between those regions that process explicit world knowledge, such as facts and events, and subserve arbitrary associations of learned information, and those regions that process implicit cognitive and motor skills, which are performed unconsciously. Ullman called the first group of regions the 'declarative' memory system and suggested that it primarily includes the hippocampus and temporal regions. The second group of regions was called the 'procedural' memory system, which primarily includes the frontal lobe and basal ganglia, with a potential role for inferior parietal regions. In a later version of his model, Ullman [2] extended the procedural network by providing a role for the cerebellum in it.

Based on neurocognitive data, Ullman [1] extended the DP model in order to account for language processing: he suggested that the procedural system subserves implicit and automatic aspects of language processing, such as processing of grammar, whereas the declarative system subserves knowledge and processing of words, i.e. the lexicon. This suggestion is compatible to experimental data that show differential processing for regularly versus irregularly inflected past tense forms (e.g. played versus kept) [3, 4]. Regular inflections such as played are thought to be processed online by the application of a linguistic rule that instructs the automatic outstripping of the suffix -ed in order to process the stem play. On the other hand, irregular forms, such as kept, are thought to be monomorphemic, occupying separate lexical entries than their stem (keep). Based on this distinction, processing of regular past tense forms should involve the procedural system, whereas processing of irregular forms should involve the declarative system. Further supporting evidence for the relevance of the DP model for language processing has 
been provided by studies on aphasics [5], as well as on people diagnosed with specific language impairment (SLI) [6]. A common finding in these studies was that people with impaired grammatical processing often had their word learning capabilities spared. Hedenius et al. [7] recently showed that a grammatically impaired group of children with SLI was unable to consolidate learning of new non-linguistic sequences, as opposed to a group of typically developing children, which showed evidence of procedural learning. This finding was interpreted as evidence for the close relationship between sequence learning and rule-based grammatical processing and as an indication that the two types of impairment may have a common underlying neurological cause. These findings suggest a close link between non-linguistic skills and grammatical processing at the brain level, which also appear independent from episodic world knowledge, including explicit language knowledge, i.e. the lexicon.

\section{The Cerebellum in Language Processing}

As already mentioned, Ullman [2] proposed a role for the cerebellum within the procedural system, which also extends to language processing. More specifically, he suggested that the cerebellum may be involved in the "error-based learning of the rules that underlie the regularities of complex structures' (p. 247). The role of the cerebellum in language processing has been supported by several studies in recent years. These include evolutionary approaches suggesting that language processing in humans evolved as a by-product of the organisation of 'syntactic' behavioural sequences for problem solving during foraging, which are largely subserved by the cerebellum [8]. De Smet et al. [9] recently reviewed a number of patient studies and unveiled the effects of cerebellar damage in syntactic processing, including difficulties in processing morphemes, as well as the role that the cerebellum may have in conditions such as aphasia, alexia, dyslexia and dysgraphia. Similarly, recent reviews [10-12] list a number of functional neuroimaging studies that suggested the activation of the cerebellum in linguistic tasks, such as word generation, object naming, stem completion and semantic judgement; importantly, they also report case studies where damage in the right cerebellum led to symptoms typical in non-fluent aphasia, such as marked agrammatism and impairment in sentence construction, leading some researchers to describe a cerebellar-induced type of aphasia [13]. The available data also suggest a functional topography of the cerebellum, with language-related tasks engaging the right lateral posterior cerebellum, along with the left prefrontal regions, for righthanded participants [14-16]. This includes areas, such as lobules VI and VII (Crus I/II) [17]. Importantly, left-handed participants appear to activate the left cerebellar homologues for the same tasks [15]. Recent findings [18] have further supported the language lateralisation within the cerebellum, suggesting, however, that it is a less lateralised region compared to other typical language areas, such as the left inferior frontal gyrus (LIFG), and that the lateralisation changes little over time before puberty.

The role of the right cerebellum in language processing was further demonstrated in a recent study by Lesage et al. [19]. Lesage and colleagues applied repetitive transcranial magnetic stimulation to the right cerebellum and caused delayed responses in subjects being tested in an auditory task where the participants had to predict linguistic input based on a specific context. The author suggested that the right cerebellum may be crucial for predicting linguistic input and linked this suggestion to the general predictive role that has been proposed for the cerebellum for motor control [20]. Similarly, Marvel and Desmond [21] administered a functional magnetic resonance imaging (fMRI) study tapping on verbal working memory in two conditions: the storage condition, where subjects were required to attend to a target letter, rehearse it silently over a delay period and match it to a probe letter, and a manipulation condition, where the subjects saw the target letter, counted two letters up and rehearsed the new letter, until the probe appeared. For manipulation versus storage, the results revealed the activation of regions that support motor planning and preparation, including the pre-motor cortex, the pre-supplementary motor area (SMA) and the bilateral superior cerebellum, as well as regions shown to have a role in working memory, such as the dorsal prefrontal cortex, the insula and the right inferior cerebellum. Marvel and Desmond suggested that their findings signified a functional specialisation within the cerebellum for verbal working memory tasks: while the inferior cerebellum is important for maintaining and updating information in working memory, the superior cerebellum remains active for the ongoing manipulation of information. Consequently, the cerebellum emerges as an important region for inner speech, which in turn supports working memory.

To summarise, the role of the cerebellum in language processing has been demonstrated in a variety of studies on both healthy and impaired populations. Among the various language-related functions of the cerebellum, the evidence linking cerebellar damage to impaired syntactic processing and agrammatism further supports the role of the cerebellum in grammatical processing as part of the procedural network. We will now turn to the predictions of the DP model for nonnative language processing, as well as the available evidence for structural changes in the bilingual brain as a function of learning a second language (L2).

\section{The DP Model in L2 Processing}

Ullman [1] suggested that the distinction between procedural and declarative aspects of language processing does not apply to late L2 learners of a language and that reliance to the 
procedural system is significantly dependent on the age of acquisition (AoA) of the L2. This is due to maturational constraints of the procedural system, which make it harder to access and utilise by late learners of an L2. To investigate the effects of AoA on L2 processing, Consonni and colleagues [22] tested balanced Italian-Friulian bilinguals and Friulian late learners of Italian (AoA, 3-6 years) in an fMRI experiment with a task combining comprehension and production: it required the generation of a verb or a noun as a response to a description, in both Friulian and Italian. Both groups were highly proficient in both languages. Their results revealed comparable brain patterns across the two groups and for both languages, with a significant distinction for processing verbs versus nouns. Consonni and colleagues concluded that, with the proficiency kept high, AoA is not a significant factor for comprehension and production in an L2; instead, high bilingual proficiency and exposure lead to the convergence of the neural substrates that process the two languages. However, it is important to note that the two groups were equally exposed to two languages since birth - the late group only received Italian linguistic instruction at the age of 3 years, while any prior naturalistic exposure to Italian cannot be ruled out. Additionally, subjects with an AoA of 3-6 years are rarely classified in the literature as 'late' learners of a language [23, 24]. The maturational constraints that Ullman suggested may not apply at that age [18]; moreover, Ullman defines as 'late' the language acquisition that takes place after puberty [2]. Therefore, the suggestions by Consonni and colleagues must be treated with caution, as far as the effects of AoA are concerned.

Although Ullman suggested that late AoA may be detrimental for the procedural acquisition of the $\mathrm{L} 2$, he also admitted that late L2 learners may become more native-like as a result of practice and experience in an L2 [2]. Recent evidence has suggested that highly proficient L2 learners of English can be native-like in demonstrating rule-based morphological processing [25]. In that study, L2 learners of English with 8 years of classroom instruction and native speakers were shown to process regular past tense inflection in English in a similar way in a self-paced reading task; more specifically, both groups showed evidence for rule-based decomposition of regularly inflected forms (played) embedded in grammatical sentences, where no such evidence was shown for irregular forms (kept). According to the Skills Acquisition Theory [26], a shift from declarative to procedural knowledge in an L2 is feasible and is dependent on successful classroom instruction. In other words, the continuous instruction of a grammatical rule leads to the proceduralisation of the rule, the application of the rule without the involvement of a declarative component. It has been suggested [27] that proceduralisation or native-like processing of grammatical rules may not be across the board, but restricted to simple concatenative rules, such as the English past tense rule. Indeed, it has been shown that L2 learners with classroom exposure do not process abstract syntactic features in an L2 [28]; however, more recent findings suggested that abstract syntactic processing can be eventually established as a result of extensive naturalistic exposure to the L2 [29].

Given the available evidence for proceduralisation of L2 processing as a matter of classroom or naturalistic exposure, it is interesting to see whether these changes in behavioural processing are accompanied by structural changes in the brain. In other words, it is worth investigating whether this 'switch' between the declarative and procedural networks is manifested in structural reorganisation of the relevant brain regions.

\section{Structural Changes in the L2 Brain}

Emerging neuroimaging evidence has suggested that the structure of the human brain can be altered as a result of learning an L2. Mechelli et al. [30] used voxel-based morphometry (VBM) analyses on the brains of native English speakers and age-matched and education-matched L2 learners of English and identified a region in the left inferior parietal cortex with significantly greater grey matter (GM) density in the L2 learners. Importantly, the GM density in that area was positively correlated to the L2 learners' proficiency level and negatively correlated to their age of L2 acquisition, suggesting a dynamic reorganisation of the brain structure in this area as a function of learning and using an L2. The importance of L2 proficiency as a predictor of structural brain changes was also demonstrated by Stein and colleagues [31], who run a longitudinal MRI study on English learners of L2 German. They acquired high-resolution anatomical brain images and a proficiency measurement from their subjects in two occasions: first, after an intense 3-week German language course (day 1), and second, after 5 months from that date (day 2). Stein and colleagues found that both subjects' proficiency and their overall GM volume had increased from days 1 to 2 . A subsequent regression analysis showed that proficiency level was a good predictor of GM density in the LIFG, an area which has also been suggested to form part of the procedural network [2]. Importantly, it appears that this kind of cortical restructuring took place even within a short amount of naturalistic exposure to $\mathrm{L} 2$.

More evidence for the relationship between L2 learning and brain structure has been provided by VBM studies investigating how the GM density in the bilingual brain is correlated to the bilinguals' performance in behavioural tasks. Grogan et al. [32] reported significant correlations between the bilinguals' performance in fluency tasks and the GM density in four areas: in the left inferior temporal lobe and bilaterally in the caudate nucleus, the cerebellum and the pre-SMA. These effects were common for their performance in both their native language (L1) and their L2, and the correlations with 
the caudate nucleus were stronger for the L2 than the L1. A subsequent study [33] suggested that vocabulary knowledge in an L2 is positively correlated to the GM density in the inferior parietal lobe, bilaterally.

Notably, some of the brain regions that have been identified as crucial for bilingual processing, more specifically the LIFG, the cerebellum and the caudate nucleus, form part of what has been described as the procedural network in the DP model [1]. Despite the importance given by Ullman to this network for the processing of grammatical rules, none of the available studies has so far attempted to correlate changes in the brain structure of bilinguals to their performance in grammatical tasks. Since there is evidence for native-like processing of the past tense rule by L2 learners [25] and also since the GM density of parts of the procedural memory system has been shown to be related to L2 performance in language tasks [32], it is worth investigating the relationship between the bilinguals' GM volume and their performance in a task tapping on grammatical processing. We, therefore, compared the structural images of the native and highly proficient non-native speakers of English, who also performed a behavioural task involving processing of the English past tense rule. This was a masked priming task with past tense forms as primes and their corresponding present tense forms as targets. We chose this task as it has already been used by studies on morphological processing in L2 and it is thought to tap into morphological rule application by leaving outside any semantic effects [34-36]. We predicted significant between-group differences in GM volume in the procedural system, as a result of its increased involvement in the proceduralisation of the $\mathrm{L} 2$. We also predicted that any bilingualism-induced effects on the procedural system will be reflected in the processing patterns of the L2 learners on the grammatical task.

Finally, although there is behavioural evidence for the effects of naturalistic exposure on L2 syntactic processing [29] and MRI evidence that even 5 months of exposure can lead to changes in cortical GM density [31], none of the available studies has investigated the effects of extensive naturalistic exposure to the structure of the bilingual brain. To investigate that, we re-ran our analysis on the L2 learners only, by using their amount of naturalistic exposure as a predictor for the GM volume across the brain.

\section{Methods}

\section{Participants}

Two participant groups took part in this study: the L2 group consisted of 17 Greek L2 learners of English (mean age, 27.5; range, 19-37; SD, 5.55). They were UK residents for an average of 4 years (range, 1-13; SD, 3.5) and started learning English after the age of 6 years (mean AoA, 7.7; SD, 2.2).
They scored $82.4 \%$ (SD, $10 \%$ ) in a proficiency test [37], which is equivalent to Mastery Proficiency. The NS group consisted of 22 English native speakers (mean age, 24.5; range, 20-38; SD, 3.9). None of the native speakers reported speaking an $\mathrm{L} 2$.

\section{Behavioural Task}

The participants were tested in a masked priming task tapping on the processing of past tense inflection in English. The experimental items that were the targets of this experiment consisted of two types of verbs: regulars and irregulars. The targets were paired with two types of primes: morphological, where the prime was the past tense form of the target (e.g. played-play), and unrelated, where the prime was orthographically, morphologically and semantically unrelated to the target (i.e. fork-play). Therefore, the experiment included four conditions: regular morphology, regular unrelated, irregular morphology and irregular unrelated. Of the four conditions, only regular morphology is predicted to elicit the application of a grammatical rule (i.e. played is decomposed into play+ ed) [2].

We used 80 English verbs as targets, along with 80 nonwords, for the purposes of the lexical decision experiment, totalling 160 trails. All targets were presented once. Forty verbs were regular, and 40 irregular. The regular and irregular verbs were paired with either a morphological or an unrelated prime in order to create the 4 conditions of the experiment, consisting of 20 pairs each. Based on CELEX [38] counts, only highly frequent verbs were used as targets. The four target lists did not significantly differ in terms of length $(F(3,76)=$ $\left.1.162, p=0.330, \eta^{2}=0.044\right)$, frequency $[F(3,76)=0.649, p=$ $\left.0.586, \eta^{2}=0.025\right]$, as assessed by CELEX, and orthographic neighbourhood density $\left[F(3,76)=0.351, p=0.789, \eta^{2}=\right.$ 0.014], as assessed by the English Lexicon Project [39]. The non-word targets in this experiment were created from real verbs, from which one letter was changed, resulting in a phonologically valid non-word (e.g. keep-*teep). Half of the non-words were created from regular verbs, and half from irregular verbs. Similar to real word targets, half of the nonword targets were paired with unrelated non-word primes and half were paired with related non-word primes, which were formed similarly to the non-word targets (e.g. *tept-*teep), creating four non-word conditions. A comparison across the eight experimental conditions (four real and four non-word) showed that they did not significantly differ in length $[F(7$, 152) $\left.=0.754, p=0.627, \eta^{2}=0.034\right]$.

Each experimental trial consisted of the following visual events: (a) a forward mask (\#\#\#\#\#\#) presented for $500 \mathrm{~ms}$, (b) the prime, presented in lowercase for $35 \mathrm{~ms}$ and (c) the target, presented in uppercase for 1,500 ms. The participants' task was to decide whether or not the target was a real word by 
pressing one of two buttons (for 'yes' and 'no' responses, respectively) as fast as possible.

\section{MRI Data Acquisition and Data Analysis}

We acquired whole-brain images with a 3.0-T Siemens MAGNETOM Trio MRI scanner with Syngo software and a 12-channel head matrix coil. These were high-resolution T1weighted MPRAGE gradient-echo 3D anatomical images collected with $176 \times 1 \mathrm{~mm}$ slices (TE, $2.52 \mathrm{~ms}$; TR, $2,020 \mathrm{~ms}$; TI, $1,100 \mathrm{~ms}$; FOV, $250 \times 250 \mathrm{~mm}^{2}$ ). The scan lasted $5 \mathrm{~min}$. Following on the analysis protocol described by Douaud et al. [40], we performed a between-group, wholebrain, voxel-by-voxel comparison of the GM volume with FSL-VBM (v1.1), an optimised VBM protocol [41] carried out with FSL [42]. Structural images were brain-extracted and GM-segmented before being registered to the Montreal Neurological Institute (MNI) 152 standard space using non-linear registration [43]. The resulting images were averaged and flipped along the $x$-axis to create a left-right symmetric, study-specific GM template. Subsequently, all native GM images were non-linearly registered to this study-specific template and 'modulated' to correct for local expansion (or contraction) due to the non-linear component of the spatial transformation. The modulated GM images were then smoothed with an isotropic Gaussian kernel with a sigma of $3 \mathrm{~mm}$. Finally, voxel-wise GLM was applied using permutation-based non-parametric testing, correcting for multiple comparisons across the brain with threshold-free cluster enhancement (TFCE) [44]. Age and gender were included in the model as covariates of no interest. This analysis created a corrected whole-brain $t$ statistical map.

\section{Results}

Our between-group analysis revealed significantly increased GM volume for the L2 group in several cerebellar areas bilaterally; the significant clusters appear in Table 1 and also
Table 1 Significant cluster peaks for non-natives $>$ natives

\begin{tabular}{lllllll}
\hline Hemi & Region & Cluster size $^{\mathrm{a}}$ & $p$ value $^{\mathrm{b}}$ & $x$ & $y$ & $z$ \\
\hline $\mathrm{R}$ & Crus I/II & 1,735 & $<0.001$ & 26 & -86 & -46 \\
$\mathrm{~L}$ & Crus I/II & 1,630 & $<0.001$ & -22 & -92 & -30 \\
$\mathrm{R}$ & $\mathrm{V}$ & 138 & 0.04 & 18 & -44 & -20 \\
\hline
\end{tabular}

All coordinates in MNI space

${ }^{\text {a }}$ Cluster size is expressed in number of $1 \mathrm{~mm}$ isotropic voxels

${ }^{\mathrm{b}}$ All $p$ values corrected for multiple comparisons with TFCE

in Fig. 1, overlaid on a standard brain template for illustrative purposes. No other significant differences were revealed. This finding suggests that learning and using an L2 can affect the structure of the cerebellum.

Since the cerebellum is part of the procedural memory network, it is, therefore, possible that increased cerebellar GM volume reflects efficient linguistic rule application by L2 learners. To investigate that, we calculated the GM volume of the cerebellar cluster that emerged from the VBM analysis; subsequently, we performed Pearson's correlations between the participants' cerebellar GM volume and their reaction times (RTs) in the behavioural task (apart from two participants who were excluded due to very low accuracy in the task). Only the RTs for trials that were accurately responded to were included in the analysis, and the two groups did not differ significantly in terms of accuracy (overall accuracy: L2, $94.5 \%$ (5\%); NS, $97 \%(3 \%) ; F(1,36)=2.422, p=0.128$, $\left.\eta^{2}=0.063\right)$. For the L2 learners, the analysis revealed a significant negative correlation between the GM volume and their $\mathrm{RTs}$ in the regular morphology condition $(r=-0.602, p=$ 0.014). This signifies that, the larger the cerebellar GM volume is, the more efficient the rule application in L2 becomes, expressed in shorter RTs. There were no significant correlations with the regular unrelated $(r=-0.340, p=0.197)$, irregular morphology $(r=-0.042, p=0.878)$ and irregular unrelated $(r=-0.240, p=0.371)$ conditions, indicating that a greater cerebellar GM is not generally beneficial for word reading or for performing lexical decisions. For the NS group,
Fig. 1 Regions with significant between-group differences in GM volume, expressed in corrected 1 $-p$ values

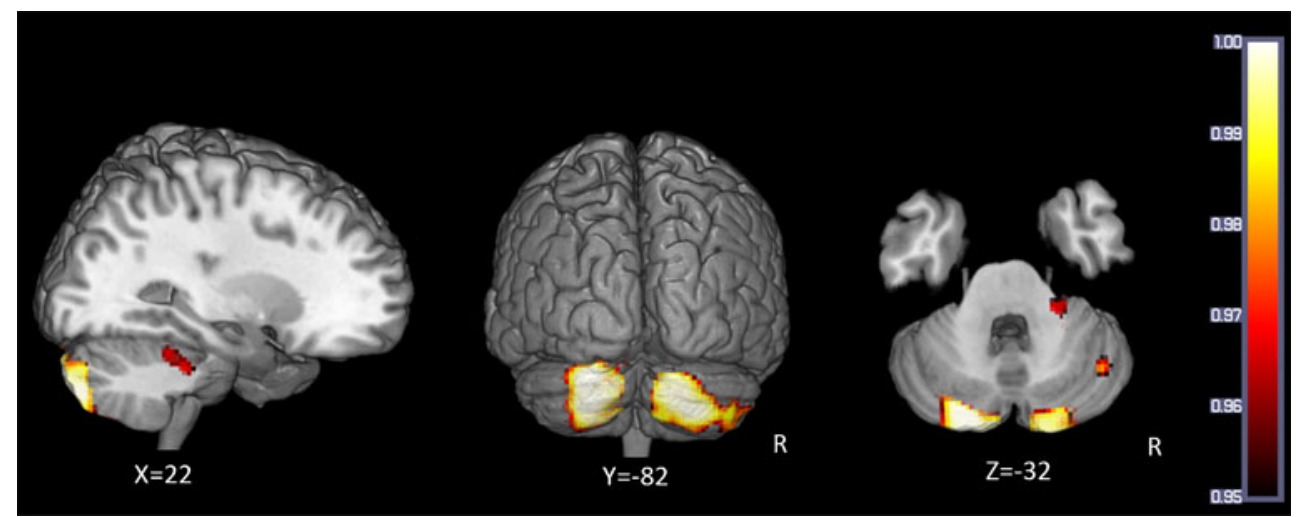



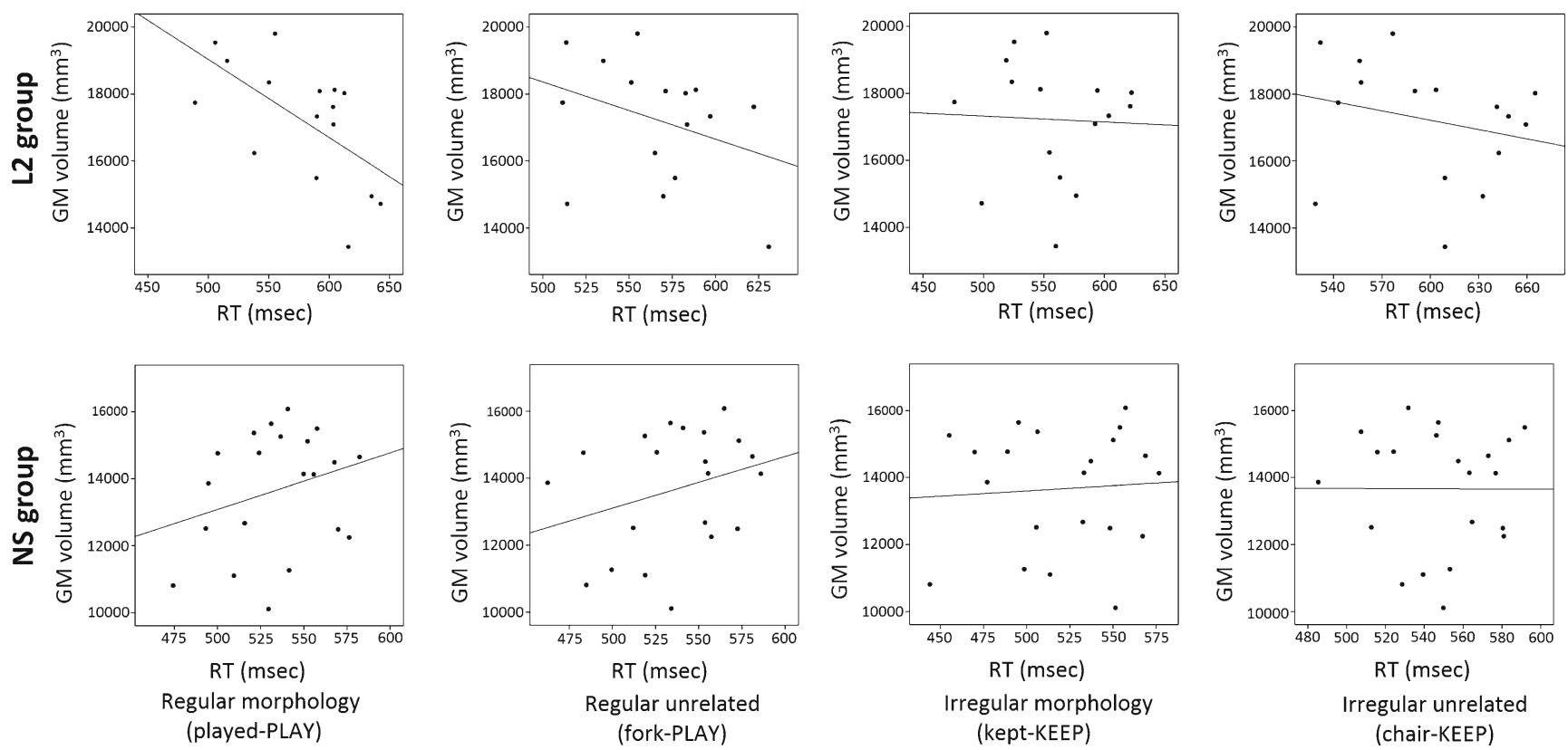

Fig. 2 Correlations between reading times and GM volume across the cerebellar regions with significantly greater GM volume for non-native speakers

the analysis revealed no significant correlations with any of the four experimental conditions (regular morphology: $r=$ $0.278, p=0.222$; regular unrelated: $r=0.293, p=0.197$; irregular morphology: $r=0.068, p=0.769$; irregular unrelated: $r=-0.004, p=0.987)$. Figure 2 shows the correlations per group for each of the four experimental conditions.

Based on recent findings that naturalistic exposure to L2 has an effect in the acquisition and processing of grammatical rules [29], we also wanted to investigate whether the amount of exposure to L2 also affects the brain structure. For this purpose, we re-ran the VBM analysis on the L2 group only and we added years of UK residence as a regressor. This analysis revealed that naturalistic exposure correlated with the GM volume in the posterior left putamen $(-26,-12,10$; $p=0.01$; cluster size, $61 \mathrm{~mm}$ isotropic voxels) and the posterior right putamen $(26,-8,6 ; p=0.02$; cluster size, $61 \mathrm{~mm}$ isotropic voxels) in the uncorrected results only. This finding calls for further investigations on the effects of naturalistic exposure on the GM.

\section{Discussion}

This study investigated whether speaking an L2 affects the structure of the brain and focused on the areas that have been proposed to subserve processing of linguistic rules [2]. Our findings can be summarised as follows: First, speaking an L2 affects the structure of the cerebellum, by leading to increased GM volume in L2 learners, compared to native speakers. According to Ullman [2], the cerebellum forms part of the procedural memory system, which subserves linguistic rule application. Second, the cerebellar GM volume of highly proficient $\mathrm{L} 2$ learners is directly related to their morphological processing in $\mathrm{L} 2$, as revealed by their performance in a masked priming language task involving the processing of the past tense rule in L2 English. Third, our data suggest that the amount of naturalistic exposure to an L2 may affect the structure of the putamen bilaterally, another structure that forms part of the procedural memory system. However, this finding did not survive the statistical correction, so it will be treated with caution. We will discuss these findings with respect to the current thinking on the role of the procedural memory system in L2 learning and processing.

\section{The Cerebellum in L2 Processing}

Our findings suggest an increase in the cerebellar GM volume for the non-native speakers of English. The cerebellum has been involved in various linguistic functions in native processing, ranging from lexical processing, such as word generation and naming, to syntactic processing [9-12]. In the L2 literature, some evidence for the importance of the cerebellum comes from Grogan and colleagues [32] who showed that GM density in the cerebellum correlates positively with L2 performance in fluency tasks. The cerebellum has been described as part of the procedural memory network [2], and as such, it is thought to underlie processing of grammatical rules, at least for native speakers of a language. Driven by recent suggestions that highly proficient L2 learners of English are nativelike in the processing of the past tense -ed rule in English [25], we investigated whether the reported increase in cerebellar GM volume is accompanied by more efficient processing of the -ed rule by L2 learners of English. Our participants took part in a masked priming experiment with regular and 
irregular past tense inflections, where the inflected forms were presented very briefly and were only subconsciously processed [34]. Our results revealed a positive correlation of the GM volume of L2 learners and their speed in processing only for the experimental condition involving regular inflection; in other words, the larger the cerebellar GM volume, the faster they processed forms like played. We propose that this finding matches the previous behavioural findings [25] and confirms the involvement of the procedural memory system in L2 morphological processing.

Since the observed increase in cerebellar GM volume is related to a behavioural measure, it can be assumed that this increase is the outcome of the experience of speaking an L2 [30] and, more specifically, of learning and applying linguistic rules of a language. These experience-based changes in the GM are common in the literature [30, 45, 46]; however, the underlying mechanism is poorly understood by means of whole-brain MRI methods. For example, Maguire et al. [46] revealed increased hippocampal volume in London taxi drivers compared to controls and attributed it to structural reorganisation within the hippocampus as a function of experience. Similarly, Mechelli et al. [30] and Stein et al. [31] report reorganisation of the cortical GM matter as a function of learning a new language; however, all three studies claim that their analyses are not appropriate to reveal the microscopic changes that take place as part of this reorganisation. GM changes can be linked to changes in the neuropil, neuronal size, arborisation or even folding [47, 48], thus it is possible that the GM changes reflect the formation of new neuronal connections to accommodate the L2 grammatical rules, although this cannot be confirmed through the VBM technique and remains open for future research.

The experience-based explanation of the structural changes and its link to the establishment of a linguistic rule is further reinforced by the fact that native speakers showed no significant correlation between the behavioural performance and the GM volume in the same cerebellar region. The important factor here is that our non-native speakers were late learners of English, i.e. they started learning the L2 after their L1 had been established, supported by the existing microarchitecture of the procedural system. Since additional languages are initially learned via a declarative route [1], the change in the cerebellar GM may reflect the proceduralisation of the L2 as an effect of practice; our subjects were highly proficient and everyday users of English for about 4 years. Therefore, the proceduralisation of the L2 may have led to the increased recruitment and usage of the procedural system, a procedure that is not necessary or relevant for the processing of the already proceduralised L1 by native speakers. Native speakers have based their linguistic processing on the existing microarchitecture- or even, the cerebellar architecture was developed as a function of first language acquisition at a very young age, and possibly before some critical period [2].
An interesting finding in our study is the fact that the cerebellum is affected bilaterally by L2 learning. There is sufficient evidence to suggest language lateralisation within the cerebellum, at least for L1 processing [9-12]. However, Berl et al. [18] suggested that, compared to more traditional language areas, such as the LIFG, the cerebellum is the least lateralised region, and its lateralisation does not increase with age, especially after the age of 4 years. Therefore, it may not be surprising that new linguistic skills that are acquired by L2 learners after the age of 6 years are less lateralised in the cerebellum. This suggestion also accounts for the findings by Consonni et al. [22], who reported overlapping activations of language brain regions for L1 and L2 acquired before the age of 6 years. These included typical left-lateralised cortical regions, as well as the right cerebellum. It is, therefore, possible that the acquisition of an L2 before or after a critical age determines the degree to which the cerebellum is recruited and lateralised; after this critical age, and according to the suggestions by Berl et al., the recruitment of the cerebellum for language learning may become less lateralised.

It is worth noting that the cerebellum did not emerge as an area affected by bilingualism in the Stein et al. [31] study, which also included late learners of L2. Stein and colleagues tested subjects upon their arrival to the L2-speaking country, and then again after 5 months of residence, and reported that the GM density in the LIFG increased parallel to the increase in their L2 proficiency. Our findings are not necessarily contradictory: the LIFG is also part of the procedural network, and it is possible that the GM density increase is a result of the intensive $\mathrm{L} 2$ instruction that these participants received during these 5 months. Thus, these findings constitute further evidence for the involvement of the procedural system in L2 learning. The fact that our analysis did not reveal any significant effects in the LIFG may be due to the different designs of the two studies: the study of Stein et al. was a longitudinal within-subjects design, whereas ours is a between-subjects design, and the former may be more sensitive in detecting cortical changes in the area. Similarly, the absence of any findings in the cerebellum in the study of Stein et al. may be due to the short exposure that these participants had in the L2; our participants had been exposed to an English-speaking environment for an average of 4 years; therefore, it is possible that the 5 months reported in the study of Stein et al. are not sufficient for detectable changes in the cerebellar structure to emerge.

Since naturalistic exposure may be an important factor for GM changes in bilinguals, it is worth discussing our finding regarding the relationship between the amount of exposure to the L2 and the size of the putamen. Our results revealed a positive correlation between the years of UK residence and the GM volume in the posterior putamen bilaterally, which, however, did not survive correction for multiple comparisons. The putamen is another structure that is part of the procedural 
memory network, and its suggested structural reorganisation over time complements our findings about the cerebellum, suggesting the increased recruitment of the procedural system as a function of learning and proceduralising an L2. However, we can only treat this uncorrected finding as an indication about how the putamen might be affected by L2 learning; it is possible that limitations in our design did not permit a stronger effect. One important aspect is the limited range of naturalistic exposure in our study, which was 12 years; Maguire et al. [46] showed a positive correlation between the hippocampal volume and the driving experience of London taxi drivers with a range of 40 years. Therefore, we suggest that future studies should be optimised to investigate this type of effects by recruiting L2 groups with a greater range and amount of naturalistic exposure. Similarly, based on recent findings that naturalistic exposure can affect syntactic processing in an L2 [29] and the acknowledged role of the cerebellum for L1 syntactic processing [13], future studies should aim to investigate for correlates between the structure of the procedural system, and especially the cerebellum, and syntactic processing in an L2.

To conclude, our study revealed that learning an L2 increases the GM volume of the cerebellum, a structure that has been suggested to form part of the procedural memory system, which subserves grammatical processing. The role of the cerebellum for grammatical processing in an L2 was further demonstrated by the positive correlation between the cerebellar GM volume and the speed in which morphologically complex forms are processed by L2 learners.

Acknowledgments This research was funded by the Economic and Social Research Council (ESRC), UK (PTA-030-2006-00359). During the write up of this manuscript, Theo Marinis was supported by an Onassis Fellowship. We would like to thank Kate Watkins and Emily Connally (University of Oxford) for their help and advice.

Conflict of Interest

We confirm that there is no conflict of interest with any third party regarding the material discussed in the manuscript

Open Access This article is distributed under the terms of the Creative Commons Attribution License which permits any use, distribution, and reproduction in any medium, provided the original author(s) and the source are credited.

\section{References}

1. Ullman MT. The neural basis of lexicon and grammar in first and second language: the declarative/procedural model. Biling Lang Cognit. 2001;4(02):717-26.

2. Ullman MT. Contributions of memory circuits to language: the declarative/procedural model. Cognition. 2004;92(1-2):231-70.

3. Marslen-Wilson WD, Tyler LK. Dissociating types of mental computation. Nature. 1997;387(6633):592-4.

4. Hartshorne JK, Ullman MT. Why girls say "holded" more than boys. Dev Sci. 2006;9(1):21-32.
5. Ullman MT, Pancheva R, Love T, Yee E, Swinney D, Hickok G. Neural correlates of lexicon and grammar: evidence from the production, reading, and judgment of inflection in aphasia. Brain Lang. 2005;93(2):185-238.

6. Ullman MT, Pierpont E. Specific language impairment is not specific to language: the procedural deficit hypothesis. Cortex. 2005;41:399433.

7. Hedenius M, Persson J, Tremblay A, Adi-Japha E, Veríssimo J, Dye $\mathrm{CD}$, et al. Grammar predicts procedural learning and consolidation deficits in children with Specific Language Impairment. Res Dev Disabil. 2011;32(6):2362-75.

8. Barton R. Embodied cognitive evolution and the cerebellum. Phil Trans Roy Soc B Biol Sci. 2012;367(1599):2097-107.

9. De Smet H, Paquier P, Verhoeven J, Mariën P. The cerebellum: its role in language and related cognitive and affective functions. Brain and Language. doi:10.1016/j.bandl.2012.11.001.

10. Baillieux H, De Smet HJ, Paquier PF, De Deyn PP, Mariën P. Cerebellar neurocognition: insights into the bottom of the brain. Clin Neurol Neurosurg. 2008;110(8):763-73.

11. Stoodley CJ. The cerebellum and cognition: evidence from functional imaging studies. Cerebellum. 2012;11(2):352-65.

12. Stoodley CJ, Schmahmann JD. Evidence for topographic organization in the cerebellum of motor control versus cognitive and affective processing. Cortex. 2010;46(7):831-44.

13. Marien P, Engelborghs S. Aphasia following cerebellar damage: fact or fallacy? J Neurolinguistics. 2000;13(2-3):145-71.

14. Ziemus B, Baumann O, Luerding R, Schlosser R, Schuierer G, Bogdahn U, et al. Impaired working-memory after cerebellar infarcts paralleled by changes in BOLD signal of a cortico-cerebellar circuit. Neuropsychologia. 2007;45(9):2016-24.

15. Jansen A, Flöel A, Van Randenborgh J, Konrad C, Rotte M, Förster A-F, et al. Crossed cerebro-cerebellar language dominance. Hum Brain Mapp. 2005;24(3):165-72.

16. Hubrich-Ungureanu P, Kaemmerer N, Henn FA, Braus DF. Lateralized organization of the cerebellum in a silent verbal fluency task: a functional magnetic resonance imaging study in healthy volunteers. Neurosci Lett. 2002;319(2):91-4.

17. Stoodley CJ, Schmahmann JD. Functional topography in the human cerebellum: a meta-analysis of neuroimaging studies. NeuroImage. 2009;44(2):489-501.

18. Berl MM, Mayo J, Parks EN, Rosenberger LR, Vanmeter J, Ratner $\mathrm{NB}$, et al. Regional differences in the developmental trajectory of lateralization of the language network. Human Brain Mapping. doi:10.1002/hbm.22179.

19. Lesage E, Morgan B, Olson A, Meyer A, Miall R. Cerebellar rTMS disrupts predictive language processing. Curr Biol. 2012;22(18):R794-5.

20. Miall R, Wolpert D. Forward models for physiological motor control. Neural Network. 1996;9(8):1265-79.

21. Marvel C, Desmond J. From storage to manipulation: how the neural correlates of verbal working memory reflect varying demands on inner speech. Brain Lang. 2012;120:42-51.

22. Consonni M, Cafiero R, Marin D. Neural convergence for language comprehension and grammatical class production in highly proficient bilinguals is independent of age of acquisition. Cortex. 2013;49:1252-8.

23. Dowens MG, Vergara M, Barber HA, Carreiras M. Morphosyntactic processing in late second-language learners. J Cogn Neurosci. 2010;22(8):1870-87.

24. Weber-Fox CM, Neville HJ. Maturational constraints on functional specializations for language processing: ERP and behavioral evidence in bilingual speakers. J Cogn Neurosci. 1996;8(3):231-56.

25. Pliatsikas C, Marinis T. Processing of regular and irregular past tense morphology in highly proficient second language learners of English: a self-paced reading study. Applied Psycholinguistics. doi:10.1017/ S0142716412000082. 
26. Lyster R, Sato M. Skill acquisition theory and the role of practice in L2 development. In: Mayo M, Guiterrez-Mangado M, Adrian M, editors. Contemporary approaches to second language acquisition. Amsterdam: John Benjamins Publishing Company; 2013. p. 71-92.

27. Clahsen H, Felser C. Grammatical processing in language learners. Appl Psycholinguistics. 2006;27(01):3-42.

28. Marinis T, Roberts L, Felser C, Clahsen H. Gaps in second language sentence processing. Stud Second Lang Acquis. 2005;27(01):53-78.

29. Pliatsikas C, Marinis T. Processing empty categories in a second language: when naturalistic exposure fills the (intermediate) gap. Biling Lang Cognit. 2013;16(01):167-82.

30. Mechelli A, Crinion J, Noppeney U, O’Doherty J, Ashburner J, Frackowiak RS, et al. Structural plasticity in the bilingual brain. Nature. 2004;431:757.

31. Stein M, Federspiel A, Koenig T, Wirth M, Strik W, Wiest R, et al. Structural plasticity in the language system related to increased second language proficiency. Cortex. 2012;48(4):458-65.

32. Grogan A, Green DW, Ali N, Crinion JT, Price CJ. Structural correlates of semantic and phonemic fluency ability in first and second languages. Cerebral Cortex. 2009;19(11):2690-8.

33. Grogan A, Parker-Jones O, Ali N, Crinion J. Structural correlates for lexical efficiency and number of languages in non-native speakers of English. Neuropsychologia. 2012;50(7):1347-52.

34. Silva R, Clahsen H. Morphologically complex words in L1 and L2 processing: evidence from masked priming experiments in English. Biling Lang Cognit. 2008;11(2):245-60.

35. Neubauer K, Clahsen H. Decomposition of inflected words in a second language. Stud Second Lang Acquis. 2009;31(03):403-35.

36. Feldman LB, Kostic A, Basnight-Brown DM, Durdevic DF, Pastizzo MJ. Morphological facilitation for regular and irregular verb formations in native and non-native speakers: little evidence for two distinct mechanisms. Biling Lang Cognit. 2010;13(02):99-118.

37. UCLES. Quick placement test (250 user CD-ROM pack). Oxford: Oxford University Press; 2001.
38. Baayen RH. The CELEX lexical database (release 2). Philadelphia: Linguistic Data; 1995.

39. Balota DA, Yap MJ, Cortese MJ, Hutchison KA, Kessler B, Loftis B, et al. The English Lexicon Project. Behav Res Meth. 2007;39(3):445-59.

40. Douaud G, Smith SM, Jenkinson M, Behrens TEJ, Johansen-Berg H, Vickers J, et al. Anatomically related grey and white matter abnormalities in adolescent-onset schizophrenia. Brain. 2007;130(9):2375-86.

41. Good CD, Johnsrude I, Ashburner J, Henson RN, Friston KJ, Frackowiak RS. Cerebral asymmetry and the effects of sex and handedness on brain structure: a voxel-based morphometric analysis of 465 normal adult human brains. NeuroImage. 2001;14(3):685700 .

42. Smith SM, Jenkinson M, Woolrich MW, Beckmann C, Behrens TEJ, Johansen-Berg $\mathrm{H}$, et al. Advances in functional and structural MR image analysis and implementation as FSL. NeuroImage. 2004;23:208-19.

43. Andersson JLR, Jenkinson M, Smith SM. Non-linear registration, aka spatial normalization. FMRIB Analysis Group Technical Reports: TR07JA2. http://www.fmrib.ox.ac.uk/analysis/techrep (2007).

44. Smith SM, Nichols TE. Threshold-free cluster enhancement: addressing problems of smoothing, threshold dependence and localisation in cluster inference. NeuroImage. 2009;44(1):83-98.

45. Draganski B, Gaser C, Busch V, Schuierer G, Bogdahn U, May A. Neuroplasticity: changes in grey matter induced by training. Nature. 2004;427(6972):311-2.

46. Maguire EA, Gadian DG, Johnsrude IS, Good CD, Ashburner J, Frackowiak RS, et al. Navigation-related structural change in the hippocampi of taxi drivers. Proc Natl Acad Sci U S A. 2000;97(8):4398-403.

47. Mechelli A, Price CJ, Friston KJ, Ashburner J. Voxel-based morphometry of the human brain: methods and applications. Curr Med Imag Rev. 2005;1(2):105-13.

48. Draganski B, May A. Training-induced structural changes in the adult human brain. Behav Brain Res. 2008;192(1):137-42. 